\title{
Intraventricular tigecycline for the treatment of shunt infection: a case in pediatrics
}

\author{
Berksu Curebal, MD, ${ }^{1}$ Nazan Dalgic, MD, ${ }^{2}$ and Banu Bayraktar, MD ${ }^{3}$ \\ Departments of ${ }^{1}$ Family Medicine, ${ }^{2}$ Pediatric Infectious Diseases, and ${ }^{3}$ Microbiology, Sisli Hamidiye Etfal Education and \\ Research Hospital, Istanbul, Turkey
}

\begin{abstract}
Ventriculoperitoneal (VP) shunt infections are seen in 3\%-17\% of patients with VP shunts. These infections may cause severe morbidity and mortality. Staphylococci are the most common cause of CSF shunt-associated infections, although gram-negative bacteria (especially multidrug-resistant [MDR] and extensive drug-resistant [XDR] bacteria) also play an important role. Due to increased antibiotic resistance, sometimes off-label usage of antibiotics is considered. Tigecycline is one of these antibiotics. It should not be used unless there are no other antibiotic treatment options available, especially in children. It belongs to the glycylcycline class of antibiotic agents and inhibits protein translation in bacteria by binding to the $30 \mathrm{~S}$ ribosomal subunit. The authors describe the case of a patient who had an XDR Klebsiella pneumoniae-positive VP shunt infection. After removal of his VP shunt, an external ventricular drain was inserted, and the patient was treated with a combination of intravenous $(1.2 \mathrm{mg} / \mathrm{kg} /$ day) and intraventricular (4 mg/day) tigecycline in addition to his meropenem (120 mg/kg/day) treatment. On the 7th day of the combined therapy, his CSF culture was sterile. Because tigecycline distribution into the tissues is not sufficient with intravenous administration, combining it with intraventricular infusion can provide new treatment methods. However, further studies are needed for its use as a treatment method in children.
\end{abstract}

https://thejns.org/doi/abs/10.3171/2018.9.PEDS18470

KEYWORDS pediatrics; tigecycline; shunt infection; intraventricular administration

$\mathrm{T}$ HE disruption of CSF flow may cause hydrocephalus, and ventriculoperitoneal (VP) shunt insertion is one of the most used treatment procedures. After shunt surgery, shunt infections are seen in 3\%-17\% of patients with a VP shunt. These infections may cause severe morbidity and mortality. After diagnosis of the infection, shunt removal, external ventricular drain (EVD) insertion, and antibiotic therapy are the basis of its management. Once the CSF is sterile, a new shunt is placed. ${ }^{11}$ Staphylococci are the most common cause of the CSF-related shunt infections, although gram-negative bacteria (especially multidrug-resistant [MDR] and extensive drug-resistant [XDR] bacteria ${ }^{8}$ ) also play an important role. The antibiotic resistance of these bacteria makes the treatment long and rather difficult. This leads to the off-label usage of new antibiotic therapies, for which there are no adequate clinical studies or literature. Tigecycline is one of these new antibiotics and belongs to the glycylcycline class. It inhibits protein translation in bacteria and blocks the entry of amino-acyl transfer RNA molecules into the A site of the ribosome by binding to the $30 \mathrm{~S}$ ribosomal subunit. It has broad-spectrum activity against MDR/XDR gram-positive and gramnegative bacteria, except for Pseudomonas spp. ${ }^{5,9}$ Due to the reported tigecycline monotherapy treatment failures, combination therapy, especially carbapenem-containing combinations, is considered an option for the treatment of MDR/XDR pathogens. ${ }^{1}$ Tigecycline usage was approved in adults by the FDA for complicated skin and skin structure infections, complicated intraabdominal infections, and community-acquired bacterial pneumonia. However, due to the observation of increased mortality under the treatment, tigecycline should not be used in children if there are other treatment options. Although tigecycline has a rapid distribution into tissues, the concentrations in CSF are low with its intravenous (IV) administration..$^{10}$ Therefore, the intraventricular (IVT) usage of tigecycline can be considerable as part of the combined therapy with a carbapenem-containing antibiotic regimen, especially against MDR Klebsi-

ABBREVIATIONS EVD = external ventricular drain; ICV = intra-cerebroventricular; IV = intravenous; IVT = intraventricular; MDR = multidrug resistant; MIC = mean inhibitory concentration; VP = ventriculoperitoneal; $\mathrm{WBC}=$ white blood cell; $\mathrm{XDR}=$ extensive drug resistant.

SUBMITTED July 25, 2018. ACCEPTED September 6, 2018.

INCLUDE WHEN CITING Published online November 9, 2018; DOI: 10.3171/2018.9.PEDS18470. 
ella pneumoniae and MDR/XDR Acinetobacter baumannii in adults. However, there have been no randomized controlled trials to evaluate the efficacy of IVT antibiotics for the treatment of central nervous system shunt infections. Especially in children, IVT antibiotics can be toxic since the brain tissue is not sufficiently mature; therefore, these drugs are only used for specific conditions, such as the unsuccessful intravenous therapy to sterilize the CSF or the existence of highly resistant organisms susceptible only to antibiotics with poor CSF penetration. There are only 3 pediatric patients with meningitis in the literature who were treated with IV tigecycline., ${ }^{2,36} \mathrm{We}$ present the first case in the literature of IV and IVT administration of tigecycline in a pediatric patient who had XDR $K$. pneumoniae due to a VP shunt infection.

\section{Case Report}

This male patient, who was born at 38 weeks' gestation with the diagnosis of congenital hydrocephalus, was admitted to a medical center with lethargy, sleepiness, and fever 20 days after his VP shunt placement. The VP shunt had been placed on the patient's postnatal 2nd day at the same center. Cranial CT showed significant dilation and septation of all ventricles. The CSF white blood cell (WBC) count was $20 / \mathrm{mm}^{3}$, protein $783 \mathrm{mg} / \mathrm{dl}$, and glucose $14 \mathrm{mg} / \mathrm{dl}$. Gram staining was negative. With the preliminary diagnoses of sepsis and shunt infection, treatment using IV cefotaxime (150-200 mg/kg/day) and IV vancomycin $(60 \mathrm{mg} / \mathrm{kg} /$ day) was started. Blood, urine, and CSF cultures were negative during the patient's follow-up, and his treatment was discontinued on its 14th day. After this treatment, the patient was discharged. In his 8th month, the patient presented to our emergency unit for the first time, with fever and unconsciousness. He had a toxic appearance and a bulging anterior fontanelle. On physical examination, his temperature was $37.1^{\circ} \mathrm{C}$, heart beat $130 \mathrm{bpm}$, and respiratory rate 45 breaths per minute. His complete blood count revealed the following: WBC count $14,660 / \mathrm{mm}^{3}$ (reference range $6000-17,000 / \mathrm{mm}^{3}$; all reference ranges are the reference ranges according to our laboratory), neutrophils $40 \%$ (reference range $15 \%-35 \%$ ), platelets 236,000 / $\mathrm{mm}^{3}$ (reference range $150,000-450,000 / \mathrm{mm}^{3}$ ), C-reactive protein $6.11 \mathrm{mg} / \mathrm{dl}($ reference $<0.5 \mathrm{mg} / \mathrm{dl}$ ), and procalcitonin $0.57 \mathrm{~g} / \mathrm{dl}$ (reference $<0.5 \mathrm{~g} / \mathrm{dl}$ ). The CSF protein was $1296 \mathrm{mg} / \mathrm{dl}$ (reference range $20-40 \mathrm{mg} / \mathrm{dl}$ ), glucose $1 \mathrm{mg} /$ dl (reference range $45-80 \mathrm{mg} / \mathrm{dl}$ ), and WBC count 2000/ $\mathrm{mm}^{3}$ (reference range $0-30 / \mathrm{mm}^{3}$ ). With these findings, it was decided that he should be hospitalized in the pediatric infectious diseases clinic, and vancomycin $(60 \mathrm{mg} / \mathrm{kg})$ and ceftriaxone $(100 \mathrm{mg} / \mathrm{kg} /$ day) were started empirically. The infected shunt was removed, and an EVD catheter was inserted. In his CSF culture, extended-spectrum $\beta$-lactamase producing $K$. pneumoniae was isolated. The patient's treatment was changed to meropenem $(120 \mathrm{mg} / \mathrm{kg} / \mathrm{day})$. He was still unconscious and had a toxic appearance. On the 10th day of his meropenem treatment, another CSF sample was taken and showed a WBC count of $7000 / \mathrm{mm}^{3}$, a glucose level of $0 \mathrm{mg} / \mathrm{dl}$, and a protein level of $2277 \mathrm{mg} / \mathrm{dl}$. XDR $K$. pneumoniae was positive in this CSF culture. ${ }^{8}$ The mean inhibitory concentration (MIC) values for cefepime, me-
TABLE 1. MIC values for the antibiotics

\begin{tabular}{lcl}
\hline \multicolumn{1}{c}{ Antibiotics } & MIC Value $(\mu \mathrm{g} / \mathrm{ml})$ & Susceptibility \\
\hline Cefepime & $>8$ & Resistant \\
\hline Meropenem & $>32$ & Resistant \\
\hline Imipenem & $>32$ & Resistant \\
\hline Ertapenem & $>32$ & Resistant \\
\hline Ciprofloxacin & $>2$ & Resistant \\
\hline Aztreonam & $>16$ & Resistant \\
\hline Piperacillin \& tazobactam & $>16 \& 4$ & Resistant \\
\hline Amikacin & $>16$ & Resistant \\
\hline Tigecycline & 1 & Susceptible \\
\hline
\end{tabular}

ropenem, imipenem, ertapenem, ciprofloxacin, aztreonam, piperacillin and tazobactam, amikacin, and tigecycline were $>8,>32,>32,>32,>2,>16,>16$ and $4,>16$, and $1 \mu \mathrm{g} / \mathrm{ml}$, respectively. The MIC results were evaluated using the recommendations of the European Committee on Antimicrobial Susceptibility Testing (EUCAST). For tigecycline, meropenem, imipenem, and ertapenem, the Etest (Oxoid) was used. For the remaining antibiotics, the BD Phoenix automated identification and susceptibility testing system (Becton Dickinson) was used. Except for tigecycline, all antibiotics were in the resistant category, meaning that the microorganisms were resistant to the obtainable serum drug levels (Table 1). IVT amikacin $(30 \mathrm{mg}$ / day) and IV tigecycline $(1.2 \mathrm{mg} / \mathrm{kg} /$ dose twice daily) were added to intravenous meropenem treatment. The patient's CSF sample was evaluated on the 6th day of the combined IV and IVT treatment and showed a WBC of $5000 / \mathrm{mm}^{3}$, a glucose level of $1 \mathrm{mg} / \mathrm{dl}$, and protein level of $1932 \mathrm{mg} /$ dl. On the 10th day of his treatment, all findings were the same, and the isolation of XDR K. pneumoniae continued. With the written permission of the patient's family, IVT amikacin treatment was discontinued, and IVT tigecycline treatment was started with the dose of $4 \mathrm{mg} /$ day. IVT tigecycline was dissolved in $10 \mathrm{ml}$ saline, and a $0.4-\mathrm{mg} /$ $\mathrm{ml}$ concentration was achieved and administered into the CSF. After every administration, CSF drainage was closed for 2 hours. At the end of the 1st week, the patient's CSF culture was negative (Table 2). IV tigecycline was discontinued on its 24th day, and IVT tigecycline was discontinued on its 14th day. The patient received a total of 34 days of meropenem therapy. No adverse effect or seizures were observed during or after the treatment, and the patient was clinically stable. After obtaining 3 consecutive negative CSF cultures, the EVD was removed, and the VP shunt was inserted. The patient was discharged.

After the 1st month of his latest discharge, the patient was admitted to our emergency department for pneumonia, and he was hospitalized. His blood, urine, and CSF cultures were taken. His blood culture was positive for $A$. baumannii, which was sensitive for colistin. The tigecycline MIC value was $16 \mu \mathrm{g} / \mathrm{ml}$. His urine and CSF cultures were sterile. His VP shunt was also evaluated by neurosurgeons and was found to function normally. In the 48th hour of treatment, the patient died of respiratory arrest. During his IVT and IV tigecycline and meropenem treatment, after his discharge and last hospitalization, no adverse effect 


\begin{tabular}{|c|c|c|c|c|c|c|}
\hline & \multirow{2}{*}{$\begin{array}{c}\text { WBCs/ } \\
\mathrm{mm}^{3}\end{array}$} & \multirow{2}{*}{$\begin{array}{l}\text { Protein } \\
(\mathrm{mg} / \mathrm{dl})\end{array}$} & \multirow{2}{*}{$\begin{array}{c}\text { Glucose } \\
\text { (mg/dl) }\end{array}$} & \multirow[b]{2}{*}{ Culture } & \multicolumn{2}{|c|}{ Treatment* $^{*}$} \\
\hline & & & & & IV & IVT \\
\hline 1st admission to our hospital & 2000 & 1296 & 1 & K. pneumoniae & - & - \\
\hline 10th day of the 1st IV treatment & 7000 & 2277 & 0 & K. pneumoniae & Cefotaxime \& vancomycin & - \\
\hline 6th day of the 1st IVT+IV combined therapy & 5000 & 1932 & 1 & K. pneumoniae & Meropenem \& tigecycline & Amikacin (30 mg/day) \\
\hline Last IVT+IV combined therapy & 3 & 45 & 50 & Negative & Meropenem \& tigecycline & Tigecycline (4 mg/day) \\
\hline
\end{tabular}

$-=$ none.

* At the time of the visit.

was observed related to the tigecycline. However, the patient did not live long enough for any adverse effects of the tigecycline treatment to be observed.

\section{Discussion}

Tigecycline is one of the new-generation antibiotics and, due to the resistance of bacteria, it is sometimes used off-label. In pediatric patients, when it is needed, it should be administered $1.2 \mathrm{mg} / \mathrm{kg}$ every 12 hours for patients between 8 and 11 years of age and $50 \mathrm{mg}$ tigecycline every 12 hours for patients between 12 and 17 years of age. Iosifidis et al. ${ }^{5}$ created a table of the pediatric cases in which tigecycline was used as treatment. They defined the type of the microorganisms, combined antibiotic therapy agents, adverse effects, and the clinical outcomes in their hospital, as well as in the literature. There are only 3 pediatric cases in the literature in which tigecycline was used for central nervous system infections. De Luca et al. ${ }^{2}$ reported on a 12-year-old boy with AIDS and meningitis due to the isolation of MDR A. baumannii on his VP shunt. The authors used IV tigecycline $3 \mathrm{mg} / \mathrm{kg} /$ day divided in 2 doses with colistin for 4 weeks, and then alone for another 4 weeks. Two weeks later, at the discontinuation of the treatment, the CSF sample showed normal results. The concentration of tigecycline in CSF was not measured. Jaspan et $a .^{6}$ described a case of vancomycin-resistant Enterococcus faecium meningitis in a 21-month-old girl who had a peripheral stem cell transplant. She was under intense myelosuppression because of acute biliniage leukemia. For the treatment of the meningitis, IVT and IV daptomycin and high-dose IV tigecycline $(2 \mathrm{mg} / \mathrm{kg}$ every 8 hours) were used to reach the maximum CSF penetration. Two days after the addition of tigecycline, the patient's CSF was sterile. The total tigecycline treatment lasted 14 days, and daptomycin was discontinued at the end of the 6th week. The CSF concentration of tigecycline was measured at $0.75 \mathrm{mg} / \mathrm{L}$. Emiroglu et al. ${ }^{3}$ recently reported the case of a 5-month-old boy with VP shunt meningitis with the isolation of carbapenem-resistant $K$. pneumoniae. IV tigecycline, $1.2 \mathrm{mg} / \mathrm{kg}$ every 12 hours, and intrathecal amikacin were added to his meropenem treatment. On the 6th day of the therapy, his CSF was sterile. He received 60 days of meropenem and 20 days of tigecycline therapy. The authors could not measure the tigecycline levels in the CSF. There are 4 cases of adults in which tigecycline was administered intraventricularly. Lauretti et al. ${ }^{7}$ described the case of a 22-year-old man who developed XDR A. bau- mannii in CSF after pituitary adenoma surgery. The authors successfully treated the patient with different doses of IVT tigecycline depending on the changes in his health condition (minimum $2 \mathrm{mg}$ /day, maximum $4 \mathrm{mg} /$ day). Fang et al. ${ }^{4}$ reported on a 50 -year-old man whose CSF culture showed XDR A. baumannii growth after the hematoma removal of the frontal and temporal lobes of the brain. In addition to his IV tigecycline and cefoperazone-sulbactam treatment, $3 \mathrm{mg} /$ day IVT tigecycline was injected. Due to the positive CSF cultures, after 6 days the dose was increased to $4 \mathrm{mg} / \mathrm{day}$. After 3 days, the CSF culture became negative. All the CSF cultures were negative for the next 3 days, and the IVT therapy was discontinued. The patient received 2 weeks of IVT tigecycline and no adverse effects were observed. Wu et al. ${ }^{12}$ described a 67 -year-old man who developed MDR K. pneumoniae meningitis after having hematoma removal and EVD placement due to cerebral hemorrhage. Based on the patient's response, the authors administered the following doses of tigecycline: 50 $\mathrm{mg}$ IV/1 mg intra-cerebroventricular (ICV) every 12 hours, $45 \mathrm{mg}$ IV/5 mg ICV every 12 hours, and $40 \mathrm{mg}$ IV/10 mg ICV every 12 hours, respectively, in combination with cotrimoxazole (sulfamethoxazole $0.4 \mathrm{~g}$, trimethoprim $0.08 \mathrm{~g}$ per pill, 2 pills per day). The level of tigecycline increased in the CSF when intravenous tigecycline was given with the increased dose. The highest concentration of tigecycline in CSF was measured with the combination of 50 $\mathrm{mg}$ IV tigecycline and $1 \mathrm{mg}$ IVT tigecycline $(2.886 \mathrm{mg} / \mathrm{L})$. Wang et al ${ }^{10}$ reported a case of a 45 -year-old man who developed MDR A. baumannii meningitis after a lumbar puncture. A dose of $10 \mathrm{mg}$ IVT tigecycline was administered every 12 hours. Six days later, the CSF cultures were negative, and the treatment was discontinued. Ours was the first pediatric case in literature in which the patient was treated with the administration of IVT tigecycline combined with IV tigecycline and meropenem. We successfully treated VP shunt-related MDR K. pneumoniae meningitis with a tigecycline IV dose of $1.2 \mathrm{mg} / \mathrm{kg} / \mathrm{day}$ and IVT dose of $4 \mathrm{mg} /$ day. However, we could not measure the tigecycline concentration in CSF. During treatment, we did not see any adverse effects of tigecycline.

\section{Conclusions}

Because the tigecycline distribution into the tissues is not sufficient with IV administration, combining it with IVT treatment can provide new treatment methods. However, the optimal therapy and optimal dose and potential 
long-term side effects of IV and IVT tigecycline are not clear, especially in children. New studies are still needed to use it as a new treatment method.

\section{References}

1. Cai Y, Bai N, Liu X, Liang B, Wang J, Wang R: Tigecycline: Alone or in combination? Infect Dis (Lond) 48:491-502, 2016

2. De Luca M, Angelino G, Calò Carducci FI, Martino A, Bernardi S, Bernaschi P, et al: Multidrug-resistant Acinetobacter baumannii infection in children. BMJ Case Rep 2011:bcr0220113807, 2011

3. Emiroglu M, Alkan G, Turk Dagi H: Tigecycline therapy in an infant for ventriculoperitoneal shunt meningitis. Pediatrics 139:e20160963, 2017

4. Fang YQ, Zhan RC, Jia W, Zhang BQ, Wang JJ: A case report of intraventricular tigecycline therapy for intracranial infection with extremely drug resistant Acinetobacter baumannii. Medicine (Baltimore) 96:e7703, 2017

5. Iosifidis E, Violaki A, Michalopoulou E, Volakli E, Diamanti $\mathrm{E}$, Koliouskas D, et al: Use of tigecycline in pediatric patients with infections predominantly due to extensively drugresistant gram-negative bacteria. J Pediatric Infect Dis Soc 6:123-128, 2017

6. Jaspan HB, Brothers AW, Campbell AJP, McGuire JK, Browd SR, Manley TJ, et al: Multidrug-resistant Enterococcus faecium meningitis in a toddler: characterization of the organism and successful treatment with intraventricular daptomycin and intravenous tigecycline. Pediatr Infect Dis J 29:379-381, 2010

7. Lauretti L, D'Alessandris QG, Fantoni M, D'Inzeo T, Fernandez E, Pallini R, et al: First reported case of intraventricular tigecycline for meningitis from extremely drug-resistant Acinetobacter baumannii. J Neurosurg 127:370-373, 2017

8. Magiorakos AP, Srinivasan A, Carey RB, Carmeli Y, Falagas ME, Giske CG, et al: Multidrug-resistant, extensively drug- resistant and pandrug-resistant bacteria: an international expert proposal for interim standard definitions for acquired resistance. Clin Microbiol Infect 18:268-281, 2012

9. Pallotto C, Fiorio M, D’Avolio A, Sgrelli A, Baldelli F, Di Perri G, et al: Cerebrospinal fluid penetration of tigecycline. Scand J Infect Dis 46:69-72, 2014

10. Wang L, Zhang J, Yu X, Wang J, Cheng L, Hu S, et al: Intrathecal injection of tigecycline in treatment of multidrugresistant Acinetobacter baumannii meningitis: a case report. Eur J Hosp Pharm 24:182-184, 2017

11. Woo PYM, Wong HT, Pu JKS, Wong WK, Wong LY, Lee MW, et al: Primary ventriculoperitoneal shunting outcomes: a multicentre clinical audit for shunt infection and its risk factors. Hong Kong Med J 22:410-419, 2016

12. Wu Y, Chen K, Zhao J, Wang Q, Zhou J: Intraventricular administration of tigecycline for the treatment of multidrugresistant bacterial meningitis after craniotomy: a case report. J Chemother 30:49-52, 2018

\section{Disclosures}

The authors report no conflict of interest concerning the materials or methods used in this study or the findings specified in this paper.

\section{Author Contributions}

Conception and design: Curebal, Dalgic. Analysis and interpretation of data: Curebal, Bayraktar. Drafting the article: Curebal, Dalgic. Critically revising the article: Dalgic, Bayraktar. Reviewed submitted version of manuscript: Dalgic. Study supervision: Dalgic, Bayraktar.

\section{Correspondence}

Berksu Curebal: Sisli Hamidiye Etfal Education and Research Hospital, Sisli, Istanbul, Turkey. berksucurebal@gmail.com. 